_ Die Altenpflege steht 2016 vor der Herausforderung, bekannte Problemstellungen wie den demografischer Wandel und den Fachkräftemangel auf den konkreten Pflegealltag herunterzubrechen und dafür Lösungen, wie den C\&S ${ }^{\circledR}$ PflegeManager, zu suchen.

2015 hat sich gezeigt, dass das anfänglich sehr zögerlich angenommene Thema der Effizienzsteigerung in der Pflegedokumentation, das Strukturmodell, immer mehr Fahrt aufgenommen hat. In der Zukunft wird sich herausstellen, inwieweit die Bemühungen, eine Reduzierung des Dokumentationsaufwandes zu erzielen, erfolgreich sind.

Es gibt einen Trend, Betreute möglichst lange im gewohnten häuslichen Umfeld verbleiben zu lassen. Politik, Sozial- und
Wohnungswirtschaft, Pflegedienste, Kommunen und auch sonstige Beteiligte sind hier gefordert. Dabei geht es neben gesetzlichen, planerischen und ökonomischen Rahmenbedingungen auch um technische Entwicklungen, die ein solches Bestreben unterstützen. Hier erweist sich gerade die Software-Industrie als Treiber, um diesen Wunsch zu realisieren. Dementsprechend bieten AAL (Alltagsunterstützende Assistenzlösungen - Ambient Assisted Living) und loTCare (Internet of Things - Internet der Dinge) speziell in der Pflege die Möglichkeit, einzeln oder durch die Verbindung beider Systeme den Wunsch nach weitest gehender Selbstständigkeit im Alter zu erfüllen. Unter AAL werden dabei technische Lösungen, sogenannte Assistenzsysteme und Dienstleistungen zur Unterstützung eines selbstbestimmten Lebens verstanden, die nicht nur im häuslichen Umfeld, sondern auch im Alltag, den Erhalt und die Förderung einer maximal möglichen Selbständigkeit zum Ziel haben. So werden sowohl Pflegeeinrichtungen, das häusliche Umfeld von Betreuten als auch das allgemeine Umfeld immer mehr zu "Smart Homes" oder "Smart Livings". Die Notwendigkeit für solche Systeme und deren Einsatzmöglichkeiten wurden im letzten Jahrzehnt in vielen europäischen Ländern erprobt und evaluiert. 2016 soll sich als das Jahr zeigen, in dem diese Lösungen in den Pflegealltag einkehren.

Nach Informationen von: $\mathrm{C} \& S^{\circledR}$ Computer und Software GmbH (www.managingcare.de)

\title{
Selbstmedikation
}

\section{Neue Studien durchleuchten Verwender}

— Die Selbstmedikation beschäftigt Pharmaindustrie, Ärzte und Apotheker gleichermaßen. Drei Studien rücken nun den Selbstmedikationspatienten in den Mittelpunkt: Zwei von ihnen beschäftigen sich mit den Persönlichkeitsstrukturen der Anwender, eine dritte soziologische Analyse geht der Frage nach, wie Abgeordnete und andere Entscheider im Gesundheitssystem den Zusammenhang von "Gesundheit und Selbstmedikation" sehen.

Prof. Dr. Elmar Brähler, Abteilung für Medizinische Psychologie und Medizinische Soziologie des Universitätsklinikums Leipzig, belegt mit seiner Untersuchung, dass soziale Persönlichkeitsmerkmale entscheidend dafür sind, wie jemand mit seiner Gesundheit und der Selbstmedikation umgeht. Sein Fazit: "Beim Patienten gibt es keinen Durchschnitt”.

Prof. Dr. Astrid Schütz vom Lehrstuhl für Persönlichkeitspsychologie und Psychologische Diagnostik der Universität Bamberg wiederum ist in ihrer Studie der Frage nachgegangen, inwieweit das Selbstbild, Bremse oder Beschleuniger beim Heilprozess eines Menschen ist. Ihr Ergebnis: Menschen mit einem statischen Blick auf sich selbst leiden stärker unter Krankheit und Belastung als andere, die sich als weniger handlungsfähig wahrnehmen und subjektiv weniger als andere der Überzeugung sind, auf die Dauer und das Ausmaß der Krankheit einwirken zu können.

Der Bamberger Soziologe Prof. Dr. Gerhard Schulze schließlich zog aus seinen Untersuchungen den Schluss: Die Patienten bestimmen immer mehr, was sie wollen, die angestammten, gesetzlich reglementierten Rollen von Arzt und Apotheker halten dem Druck der Veränderung kaum noch stand Die Zukunft des Gesundheitswesens benötige ein neues Gesundheitsverständnis, eine verstärkte persönliche Gesundheitskompetenz und dadurch eine veränderte Arzt-Apotheker-Patientenkommunikation.

\section{Autotransfusion \\ Intuitive Bedienung}

— Das neue System zur maschinellen Autotransfusion von Fresenius Kabi Deutschland, CATSmart, soll eine verbesserte Qualität und optimierter Handhabung bieten. Durch die neuen Waschprogramme steht aufbereitetes Blut schnell und mit konstantem Hämatokrit zur Verfügung. Der Touchscreen ermöglicht im Zusammenspiel mit dem neuen Farbkonzept eine intuitive Bedienung. So können Einstellungen zügig vorgenommen werden. Die Aufbereitung von Blut findet sehr leise statt und die maschinelle Autotransfusion ist höhenverstellbar. Außerdem ermöglicht das neue System den Patienten bei Operationen oder hohem Blutverlust eine rasche Bereitstellung eigener Erythrozyten. So reduziert sich die Notwendigkeit einer Transfusion von Fremdblut.

www.fresenius-kabi.de 